\title{
KANDINSKY UND DIE TRADITION
}

\author{
Klaus Lankheit zum 65. Geburtstag \\ Von Peter Anselm Riedl
}

Seit Klaus Lankheit 1951 seinen aufsehenerregenden Aufsatz „Die Frühromantik und die Grundlagen der ,gegenstandslosen 'Malerei" veröffentlicht hat, gehört das Wissen um Zusammenhänge zwischen den Pioniertaten Kandinskys und der deutschen Literatur, Philosophie und Malerei um 1800 weithin zum Fundus des kunstgeschichtlich Informierten. Die von Lankheit gewonnenen Einsichten sind mittlerweile durch zahlreiche Belege erhärtet und um wichtige Erkenntnisse vermehrt worden. Namentlich Otto Stelzers 1964 publizierte „Vorgeschichte der abstrakten Kunst" bescherte eine überraschende Fülle neuen Materials und half eine von Lankheit noch nicht erörterte Auffassung zu stiften: nämlich die einer Kontinuität, einer inneren Schlüssigkeit der Entfaltung bestimmter Möglichkeiten der Kunsttheorie und -praxis im Laufe des 19. Jahrhunderts. Nicht nur das komplexe Bild einer von verschiedenen Quellen gespeisten und an individuellen Nuancen reichen Überlieferung trat in den Blick; auch der Gedanke an einen direkten Brückenschlag in die Vergangenheit bot sich an, ließ sich doch in der ungemein belesenen und mit manchen Vorstellungen der deutschen Romantik solidarischen Marianne von Werefkin eine für Kandinsky und den Münchener Künstlerkreis bedeutsame Schlüsselfigur vermuten.

Nun erhielt die Kandinsky-Diskussion in den letzten Jahren kritische Impulse, die auf differente Weise den historischen Ansatz verschoben. Auslösend waren in allen hier gemeinten Fällen die esoterischen und irrationalen Elemente in Kandinskys literarischen und bildnerischen Äußerungen, Elemente also, die einerseits den Rückbezug auf die Romantik in hohem Maße stützen und die andererseits für ein aktuelles Verständnis Herausforderungen besonderer Art repräsentieren.

Werner Hofmann etwa sucht in seinen "Grundlagen der modernen Kunst" - wie er ausdrücklich versichert, nicht „um Kandinskys schöpferischen Rang zu schmälern, sondern um ihn zu proklamieren" - die rationalen Schaffenskomponenten energisch aufzuwerten und der Absicht, dem Mißtrauen des Publikums gegenüber der neuen Kunstsprache durch einen Pakt mit dem Kosmos zu begegnen, mehr Gewicht zuzumessen als philosophischen und theosophischen Neigungen. Demütiges Bekenntnis zu übergreifenden Mächten in der Funktion einer Schutzattitüde also, berechnetes Chiffrieren der bildnerischen Anliegen! Bemerkenswerterweise versteht Hofmann die For-

*) Festvortrag zur Jahresfeier der Universitäts-Gesellschaft am 30. November 1977 
mensprache der dynamisch-expressiven Phase Kandinskys mehr im Sinne einer inszenierten Vorläufigkeit als einer genuinen Spontaneität und sieht sie in der Nachfolge der improvisierenden Handschrift früherer Epochen, insonderheit der „,confused mode of execution" eines Crane. Der Grundtendenz der Interpretation entsprechend, wird den 1914 formulierten Sätzen: „Ich will keine Musik malen. Ich will keine Seelenzustände malen" hoher Aussagewert zuerkannt. Wenn Hofmann auch die Vieldeutigkeit Kandinsky relativiert: Grundsätzlich geleugnet wird sie keineswegs.

Einen anderen Versuch rationalisierender Deutung stellt L. D. Ettlingers Charlton Lecture „Kandinsky's ,At Rest" " von 1961 dar. Die Theosophie wird als Quelle eines bei Kandinsky nicht seltenen „pretentious nonsense ${ }^{\text {et }}$ angegeben, der Wahrnehmungspsychologie eine tragende Rolle eingeräumt. Berührungen mit den Theorien Francis Galtons und Ernst Machs, später mit der Gestaltpsychologie seien zu unterstellen; gerade die Gestaltpsychologie sei zur wissenschaftlichen Rückendeckung des abstrakten Verfahrens prädestiniert gewesen. Auch hier klingt also - wenngleich anders motiviert - der Gedanke an ein Sich-Verschanzen an.

Im Gegensatz zu den genannten Autoren verwies Sixten Ringbom in einem 1966 im ,Journal of the Warburg and Courtauld Institutes" veröffentlichten Aufsatz „Art in the ,Epoch of the Great Spiritual': Occult Elements in the Early Theory of Abstract Painting "e auf die dominierende Bedeutung okkulter, namentlich theosophischer Elemente für die Kunsttheorie Kandinskys. In einem wenig später erschienenen Buch „The Sounding Cosmos - A Study in the Spiritualism of Kandinsky and the Genesis of Abstract Painting" beharrt Ringbom auf den vorher dargelegten Gedanken und breitet eine solche Fülle neuer Belege aus, daß sich auch skeptische Leser des ersten Artikels zu einer mindest teilweisen Kapitulation gezwungen sehen müssen.

Nun schließt die - prinzipiell ohnedies unbestreitbare - Existenz hermetischen Vorstellungsgutes Auslegungen im Sinne Hofmanns oder Ettlingers nicht aus. Die Frage ist die nach dem Anteil solcher Vorstellungen am geistigen Gesamtsystem (sofern man hier von so etwas wie einem System sprechen darf!) und besonders die nach Aufrichtigkeit und Intensitätsgrad der Überzeugung Kandinskys. Eine zweite Frage ist die nach der Qualität des Traditionsbezuges des Künstlers. Um zunächst diese zweite Frage zu prüfen: Welches wären die Gemeinsamkeiten einer bereits um 1800 diagnostizierbaren Auffassung und der Haltung Wassily Kandinskys? Im Sinne der Argumentationskette Lankheits folgende: die Ablehnung des von außen diktierten Gegenstandes (in der Konsequenz die Verabschiedung des Gegenstandes überhaupt); das Beharren auf der schöpferischen Kraft des Individuums; die Idee der Verwandtschaft der Künste und eines Sonderranges der Musik; der antirationalistische Glaube an das Walten einer übergreifenden Ordnung, in die Natur und Geist hineingebettet sind und die sich in allen Schöpfungen manifestiert.

Oft schon sind nach dem Vorbild Lankheits die Passagen aus Tiecks „Franz Sternbalds Wanderungen" zitiert worden, in denen sich geradezu programmatisch ein neues Verhältnis zur Kunst ankündigt. $\mathrm{Da}$ heißt es angesichts des Abendhimmels: „... wenn ihr Maler mir dergleichen darstellen könntet, so wollte ich euch oft eure beweglichen Historien, eure leidenschaftlichen und verwirrten Darstellungen mit allen unzähligen Figuren erlassen. Meine Seele sollte sich an diesen grellen Farben ohne Zusammenhang, an diesen mit Gold ausgelegten Luftbildern ergötzen und genügen, ich würde da Handlung, Leidenschaft, Komposition und alles gern vermissen ... O, mein Freund, wenn ihr doch diese wunderlich Musik, die der Himmel heute dichtet, in eure 
Malerei hineinlocken könntet! Aber euch fehlen Farben, und Bedeutung im gewöhnlichen Sinne ist leider die Bedingung eurer Kunst". Und an einer anderen Stelle: „Hier ist keine Handlung, kein Ideal, nur Schimmer und verworrene Gestalten ... Aber wenn Ihr dies Gemälde sähet, würdet Ihr Euch nicht mit mächtiger Empfindung in den Gegenstand hineinsehen? Würde er die übrige Kunst und Natur nicht auf eine Zeitlang aus Eurem Gedächtnisse hinwegrücken, und was wollt Ihr mehr? Diese Stimmung würde dann so wie jetzt Euer ganzes Inneres durchaus ausfüllen. Euch bliebe nichts zu wünschen übrig, und doch wäre es nichts weiter als ein künstliches, fast tändelndes Spiel der Farben. Und doch ist es Handlung, Ideal, Vollendung ..." Außenwelt wird also auf subjektives Empfinden und synästhetisches Erleben zurückbezogen (man prüfe nur die Aufforderung, die Musik, die der Himmel dichtet, in die Malerei hineinzulocken, und die Bemerkung, daß solchermaßen Ideal und Vollendung erreichbar seien!). Was das Infragestellen des Gegenständlichen angeht, wird eine andere Stelle des „Sternbald"e noch deutlicher: „,Welcher Inhalt soll denn in meinem Liede sein? , ,Welcher du willst, ... wenn es dir recht ist, gar keiner; wir sind mit allem zufrieden, wenn es dir nur gemütlich ist, warum soll eben Inhalt den Inhalt eines Gedichtes ausmachen "“

Die Koppelung der Vorstellung von einer autonomen Kunst, die auf das subjektive Gefühl von Urheber und Rezipienten gegründet ist, mit der Erwartung synästhetischen Erfahrungsvermögens läßt sich, wie Stelzer gezeigt hat, bis in spätere Phasen der Romantik nachweisen. Es mag vor allem Stifters Beschreibung der Sonnenfinsternis von 1842 in Wien interessieren, in der es von alten Deskriptionen dieses Naturphänomens heißt: „Sie können nur das Geschehene malen, aber schlecht, das Gefühlte noch schlechter, aber gar nicht die namenlos tragische Musik von Farben und Lichtern, die durch den ganzen Himmel liegt - ein Requiem, ein Dies irae ... Könnte man nicht auch durch Gleichzeitigkeit und Aufeinanderfolge von Lichtern und Farben ebensogut eine Musik für das Auge wie durch Töne für das Ohr ersinnen?"

Als Gegenexempel einer solchen Beschreibung seien einige Sätze aus Kandinskys „Rückblick" zitiert: „Die Sonne schmelzt ganz Moskau zu einem Fleck zusammen, der wie eine tolle Tuba das ganze Innere, die ganze Seele in Vibration versetzt. Nein, nicht diese rote Einheitlichkeit ist die schönste Stunde! Das ist nur der Schlußakkord der Symphonie, die jede Farbe zum höchsten Leben bringt, die ganz Moskau wie das fff eines Riesenorchesters klingen läßt und zwingt. Rosa, lila, gelbe, weiße, blaue, pistaziengrüne, flammenrote Häuser, Kirchen - jede ein selbständiges Lied - der rasend grüne Rasen, die tiefer brummenden Bäume, oder der mit tausend Stimmen singende Schnee, oder das Allegretto der kahlen Äste, der rote, steife, schweigsame Ring der Kremlmauer und darüber, alles überragend, wie ein Triumphgeschrei, wie ein sich vergessendes Halleluja der weiße, lange, zierlich ernste Strich des Iwan-Weliky-Glockenturmes... Diese Stunde zu malen, dachte ich mir als das unmöglichste und höchste Glück des Künstlers."

Synästhetische Phänomene sind freilich nicht erst seit der Zeit um 1800 geläufig, man denke nur an Bertrand Castels Bemühungen um ein "Farbenklavier" in der ersten Hälfte des achtzehnten Jahrhunderts. Aber die Rückbindung synästhetischer Wahrnehmung an das Individualgefühl und der Versuch, darauf eine Kunstauffassung zu gründen, bedurften spezifischer Voraussetzungen. Wenn Goethe darüber meditiert, daß man „die Farbe sogar zu fühlen glaubt", ja, daß sie den Geschmacksinn anspreche („blau wird alkalisch, gelbrot sauer schmecken"), wenn Philipp Otto Runge der Idee 
einer "abstrakten, malerischen phantastisch-musikalischen Dichtung mit Chören" nachhängt, dann wird der utopische Charakter solcher Gedanken von durchaus kritischen Konzeptionen hinterfangen. Goethes Farbenlehre und Runges farbtheoretische Untersuchungen sind frühe Marksteine einer zukunftsreichen Entwicklung. Die physiologischen und psychologischen Erkenntnisse Goethes reflektieren sich mannigfach in Kandinskys „Über das Geistige in der Kunst"; allein ein Satz wie: „Auch ohne Bezug auf Gestalt reizen die Farben" läßt die Tragweite der Goetheschen Einsichten in Richtung auf die Möglichkeit einer Verabsolutierung des Kolorits ahnen.

Bei Kandinsky gewinnen synästhetische Überlegungen einen eigentümlich emphatischen und zugleich esoterischen Charakter. „Die Form selbst", heißt es in „Über das Geistige in der Kunst", ,wenn sie auch ganz abstrakt ist und einer geometrischen gleicht, hat ihren innern Klang, ist ein geistiges Wesen mit Eigenschaften, die mit dieser Form identisch sind. Ein Dreieck (ohne die nähere Bezeichnung, ob es spitz, flach, gleichseitig ist) ist ein derartiges Wesen mit dem ihm allein eigenen geistigen Parfüm. In Verbindung mit anderen Formen differenziert sich dieses Parfüm, bekommt beiklingende Nuancen, bleibt aber im Grunde unveränderlich, wie der Duft der Rose, der niemals mit dem des Veilchens verwechselt werden kann. Ebenso Kreis, Quadrat und alle anderen möglichen Formen." Behalten wir die Worte ,innerer Klang" und „geistiges Wesen" in Erinnerung und verweilen wir zunächst bei der Tatsache, daß Kandinsky ohne weiteres von der Sprachkraft einer auch gegenstandsunabhängigen Form ausgeht, in der Theorie und in der künstlerischen Praxis. Gerade den Schritt von theoretischen Ansätzen zur gestalterischen Verwirklichung hat die Romantik nicht getan. Bei aller Eigenenergie der chromatischen Fügung von Runges „Morgen" oder der formalen Struktur von Friedrichs "Gescheiterter Hoffnung" ist das Figurative jeweils ein unverzichtbarer Wert. Goethes zorniger, von Sulpiz Boisserée überlieferter Ausfall: „Maler Friedrich seine Bilder können ebenso gut auf den Kopf gesehen werden" zielt wohl weniger auf eine inhärente Ungegenständlichkeit als auf unorthodoxe Kompositionseigenarten.

Auch die frühen, vor allem von Stelzer nachgewiesenen Beispiele widerlegen nicht den behaupteten Sachverhalt. Die Illustrationen Laurence Sternes in der Erstausgabe des "Tristram Shandy" von 1761 sind ironische Korrelate des Textlabyrinths: Das polyfokale farbige „Marmorblatt" wird als „,buntes Sinnbild" des Werkes ausgegeben, das schwarze Blatt als ,mystische" Verhüllung von „Meinungen, Abhandlungen und neuen Wahrheiten". So originell und frappierend die optischen Kommentare sein mögen, sie sind nicht mehr als schrullige Aperçus, die außerhalb des literarischen Zusammenhanges weder bestehen können noch wollen. William Turners 1843 datiertes Gemälde "Light and Colour" hat zum einen betont demonstrativen Charakter (ein Nebentitel "Göthes Theory" spielt auf eine bestimmte Plusfarbenfolge an) und verzichtet zum anderen nicht auf - wenn auch rezessive - literarisch-figurative Elemente (weitere Nebentitel lauten: „Morgen nach der Sintflut" Cozens' „Blotting“-Skizzen stehen letztlich in der „Macchia"-Tradition, und Justinus Kerners „Klecksographien" - Vorläufer der Rorschach-Testbilder - können sich auf den alten Concetto der vom Zufall erzeugten, vieldeutigen Einseh-Figurationen berufen (Leonardo da Vinci!).

Zugegeben: Solche protoromantische bis spätromantische Manifestationen haben in der Vorgeschichte der abstrakten Kunst ihren Stellenwert, nur hat man sich vor einer einseitig aktualisierenden Deutung zu hüten. Wahr ist, daß den theoretischen Exkur- 
sen der Zeit um 1800 keine in sich schlüssige bildnerische Realisation entspricht und daß der nicht selten begegnende Begriff ,abstrakt" nicht einfach Ungegenständlichkeit impliziert, sondern mehr auf Stilisierung, Phantasieschöpfung, Märchenhaftigkeit hinzielt.

Aber nehmen wir ein Argument auf, das in den vorhin zitierten Sätzen Kandinskys mitschwingt und in der Formel vom „Prinzip der inneren Notwendigkeit" seinen prägnantesten Ausdruck findet. Innere Notwendigkeit: Das meint Autorität der künstlerischen Botschaft im Zeichen einer Gesetzlichkeit, die den Künstler auf besondere Weise mit der Natur, mit dem Kosmos verbindet, meint Innerlichkeit als derart legitimierte Leitinstanz künstlerischen Schaffens. Man denkt an Caspar David Friedrichs Aufforderung: „Der Maler soll nicht bloß malen, was er vor sich sieht, sondern auch, was er in sich sieht. Sieht er aber nichts in sich, so unterlasse er auch zu malen, was er vor sich sieht." Oder an Novalis, der in dem Romanfragment "Die Lehrlinge zu Sais" von der allenthalben vorfindlichen ,großen Chiffernschrift" erzählt, von der geheimnisvollen Verwandlungskraft des inneren Blickes, von dem Durst nach der "Weltsee$1 e^{e "}$, der „Sehnsucht nach dem Zerfließen"e; „... auf Flügeln, Eierschalen, in Wolken, im Schnee, in Kristallen und in Steinbildungen, auf gefrierenden Wassern, im Innern und $\ddot{A}$ ußern der Gebirge, der Pflanzen, der Tiere, der Menschen, in den Lichtern des Himmels, auf berührten und gestrichenen Scheiben von Pech und Glas, in den Feilspänen um den Magnet her" bemerkt er die Chiffren einer die Wege des Menschen bestimmenden Macht.

Bei Kandinsky liest sich das so: „Alles ,Tote erzitterte. Nicht nur die bedichteten Sterne, Mond, Wälder, Blumen, sondern auch ein im Aschenbecher liegender Stummel, ein auf der Straße aus der Pfütze blickender, geduldiger weißer Hosenknopf, ein fügsames Stückchen Baumrinde, das eine Ameise im starken Gebiß zu unbestimmten und wichtigen Zwecken durch das hohe Gras zieht, ein Kalenderblatt, nach dem sich die bewußte Hand ausstreckt, die es aus der warmen Geselligkeit mit den noch im Block bleibenden Mitblättern gewaltsam herausreißt - alles zeigte mir sein Gesicht, sein innerstes Wesen, die geheime Seele, die öfter schweigt als spricht. So wurde für mich jeder ruhende und jeder bewegte Punkt (= Linie) ebenso lebendig und offenbarte mir seine Seele. Das war für mich genug, um mit meinem ganzen Wesen, mit meinen sämtlichen Sinnen die Möglichkeit und das Dasein der Kunst zu ,begreifen', die heute im Gegensatz zur,Gegenständlichen' die ,Abstrakte genannt wird." Und an anderer Stelle: „Ein Künstler, welcher in der .... Nachahmung der Naturerscheinungen kein Ziel für sich sieht und ein Schöpfer ist, welcher seine innere Welt zum Ausdruck bringen will ..."; schließlich: „Werkschöpfung ist Weltschöpfung".

Das sind - beim Romantiker wie bei Kandinsky - anspruchsvolle und dunkle Worte. „Geheimes durch Geheimes ausdrücken“e: Dieses Rezept Kandinskys scheint das Schaffen der Kontrollierbarkeit zu entheben und im Raum der Kryptästhesie anzusiedeln. Mit guten Gründen hat man auf Diderots „Soyez ténébreux!" verwiesen, auf Friedrich Schlegels „Noch viel verborgene Unverständlichkeit wird ausbrechen müs-

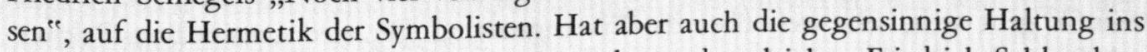
Bewußtsein gehoben, die sich in einer Bemerkung des gleichen Friedrich Schlegel zu beißender Schärfe steigert: „Es gibt Schriftsteller, die Unbedingtes trinken wie Wasser, und Bücher, wo selbst die Hunde sich aufs Unendliche beziehen."

Wir haben auf das Problem des Rätselvollen bei Kandinsky bald zurückzukommen, müssen an dieser Stelle indessen festhalten, daß die Vorstellung einer inneren Notwen- 
digkeit und einer Verschwisterung von Kunst und Natur auch in Goethes Denken eine wichtige Position behauptet. Der Künstler als Vollstrecker der Ideen der Natur, die Existenz einer Natur und Kunst umfassenden Wahrheit und die Forderung, das Kunstwerk müsse eine für seinen Urheber charakteristische, innere Form besitzen und gleichsam eine Spielart des Naturwirkens repräsentieren: Diese Themen Goethes waren für die Romantik adaptierbar und sollten, wie später zu erläutern sein wird, noch eine Konsequenz besonderer Art zeitigen.

Goethesche Elemente finden sich, originell modifiziert, in der Kunsttheorie eines Denkers des fortgeschrittenen neunzehnten Jahrhunderts, dessen Schriften Kandinsky mit Sicherheit bekannt gewesen sind. Konrad Fiedler - er ist hier gemeint - hat das Prinzip der inneren Notwendigkeit in seinem Sinne formuliert und der Schöpferkraft des Künstlers eine dominierende Funktion zugemessen: „Notwendigkeit kann einem Kunstwerk nicht von außen kommen, etwa durch Vergleichung; sie kann nur das Resultat des inneren Gleichgewichts der Elemente sein, aus denen das Kunstwerk besteht"; und: Es gebe ein „Reich der Sichtbarkeit, in welches nicht mehr das Auge, sondern nur die Sichtbares gestaltende Tätigkeit vordringen kann".

Die aufgewiesenen Entsprechungen und Traditionslinien sind durchaus geeignet, bestimmte Züge in Denken und Handeln Kandinskys zu erklären. Auf Goethe nimmt der Künstler mehrfach Bezug, Fiedlers Theorien waren ihm erwiesenermaßen zugänglich, romantisches Gedankengut konnte ihn auf mehreren Wegen erreichen. Einmal über Marianne von Werefkin, die in ihren Tagebüchern so bemerkenswerte Erkenntnisse fixiert hat wie diese: „Das große Verdienst der Romantik ist es, daß sie den ersten Stein für die Zukunft gelegt hat ... Die Kunst der Zukunft ist die emotionale Kunst", oder : „Alle Leiden verwandeln sich in mir in Töne und Farben." Kandinskys Verhältnis zur Werefkin und zu Jawlensky war allerdings erst seit 1908 etwas enger und kaum jemals besonders herzlich. Sodann über die bedeutende Nebenlinie des französischen Symbolismus: Kandinsky beruft sich in "Über das Geistige in der Kunst" in wichtigem Zusammenhang mit Nachdruck auf Maurice Maeterlinck. Werke deutscher romantischer Dichter und Philosophen standen um 1900 in Frankreich so hoch im Kurs, daß man von einem Geistesleben à la mode germanique sprechen konnte. Die Rolle der intuitiven und antirationalistischen Erkenntnislehre Henri Bergsons sei hier nur mit einem Wort erwähnt. Auch der russische Symbolismus, bereits vor Kandinskys Übersiedlung nach München im Jahre 1896 in voller Blüte, transportierte deutschromantische Gedanken. Bleibt schließlich noch die eigentlich nächstliegende Begegnungsmöglichkeit, nämlich die der direkten Lektüre. Werke deutscher Dichter und Philosophen der Romantik wurden im Zeichen eines neu erwachenden Interesses um 1900 wiederaufgelegt. Auf der Jahrhundertausstellung des Jahres 1906 präsentierte Hugo von Tschudi überdies dem Vergessen entrissene Gemälde romantischer Künstler. Nun hat Kandinsky in einem Brief an Will Grohmann später (nämlich 1925) zwar ein respektgebietendes Verständnis für die Romantik dokumentiert: „Ich will nicht mit Tränen um der Tränen willen malen, ich mag das Süße wirklich nicht, aber die Romantik geht weit, sehr weit über die Tränengrenzen." Für eine unmittelbare Bekanntschaft mit den Quellen solch bewundernder Einsicht fehlt indessen der schlüssige Beweis.

Die Frage spitzt sich also zu: Hat Kandinsky von verschiedenen Seiten Anregungen akzeptiert und synkretistisch vermengt? Hat ihn Wahlverwandtschaft bestimmte Traditionselemente aufgreifen und mit anderen, primär individuellen legieren lassen? $\mathrm{Ha}$ ben näher definierbare Einwirkungen seine theoretischen Überlegungen und künstleri- 
schen Entscheidungen mitbestimmt, Einwirkungen, die sich von den bislang genannten Wurzeln her nicht oder nur unzulänglich verstehen lassen und denen unter Umständen selber eine Mittlerrolle zukommt?

Zu den merkwürdigsten Eigenschaften der Schriften Kandinskys - namentlich der zwischen 1910 und 1914 verfaßten - gehört der erregte, oft genug in mystisch-messianisches Pathos umschlagende Ton. „Unsere Seele", heißt es in der Einleitung von „Über das Geistige in der Kunst", „die nach der langen materialistischen Periode erst im Anfang des Erwachens ist, birgt in sich Keime der Verzweiflung des Nichtglaubens, des Ziel- und Zwecklosen. Der ganze Alpdruck der materialistischen Anschauungen, welche aus dem Leben des Weltalls ein böses zweckloses Spiel gemacht haben, ist noch nicht vorbei. Die erwachende Seele ist noch stark unter dem Eindruck dieses Alpdruckes ... Nach der Periode der materialistischen Versuchung, welcher die Seele scheinbar unterlag und welche sie doch als eine böse Versuchung abschüttelt, kommt die Seele, durch Kampf und Leiden verfeinert, empor."

Die Vorstellung einer dramatischen Polarität von Materialistisch-Positivistischem einerseits und geläuterter Innerlichkeit andererseits kehrt in immer neuen Wendungen wieder. Mit sektiererisch anmutender Inbrunst wird das Heraufziehen einer Ära des großen Geistigen beschworen, in der sich das Prinzip der inneren Notwendigkeit auch den jetzt noch Blinden erschließt; wird die Mission des Künstlers als eines Protagonisten geistiger Erneuerung geschildert. Worte wie Chaos und Kosmos, Seele und Wesen werden viele Male bemüht, Künstler und Denker verschiedenster Observanz als Kronzeugen aufgerufen. In der Tat: Diskurse und Appelle, die den Leser reichlich ratlos machen und von einem fremd gewordenen geistigen Klima zeugen! Aber ist das leichthin geschrieben, ist es esoterische Maske, hinter der sich minder Anspruchswürdiges verbirgt? In der Einleitung für die Kölner Ausstellung im Jahre 1914 finden sich Passagen, die solchen Verdacht entkräften könnten. Da heißt es zunächst: „Die Entstehung meines Werkes ist kosmischen Charakters. Der Urheber des Werkes ist also der Geist. Das Werk existiert also abstrakt vor seiner Verkörperung, die den menschlichen Sinnen das Werk zugänglich macht. Für diese also notwendige Verkörperung ist jedes Mittel recht. Also die Logik ebenso wie die Intuition"; später: „Und das ist die kosmische Tragik, in der das Menschliche nur ein Klang ist ... und in der das Zentrum in eine Sphäre verschoben wird, die sich dem Göttlichen nähert. Diese Ausdrücke muß man vorsichtig brauchen und nicht mit ihnen spielen. Ich wende sie hier aber bewußt an." Kandinsky sichert sich mithin ausdrücklich gegen die Unterstellung ab, leichtfertig mit großen Worten umzugehen. Und er verweist auf die Zulässigkeit der Koppelung von Intuition und Logik, beharrt also nicht auf der Alleinkompetenz des Gefühls bei der Produktion eines dem Gesetz der inneren Notwendigkeit unterworfenen Werkes. In den Zusammenhang des Kölner Ausstellungstextes gehören auch die Versicherungen: „Ich will keine Musik malen. Ich will keine Seelenzustände malen. Ich will nicht farbig oder unfarbig malen. Ich will in der Harmonie der Meisterwerke der vergangenen Zeit keinen Punkt ändern, bekämpfen oder umwerfen. Ich will nicht der Zukunft ihren richtigen Weg weisen."

Man könnte aus alledem Rückzugsbereitschaft gegenüber dem kurz vorher in ,Über das Geistige in der Kunst" Verkündetem herauslesen, und vielleicht war ein gewisser Wille zur Korrektur auch am Werke. Aber man wird zugeben müssen, daß entscheidende Grundpositionen gewahrt bleiben und daß Widersprüche der genannten Art auch in der älteren Schrift begegnen. 
Schließlich ist in „Über das Geistige in der Kunst" ausgesprochen, daß eine Kunst bei der anderen lernen müsse, wie diese mit ihren Mitteln umgeht, ,um dann ihre eigenen Mittel prinzipiell gleich zu behandeln, d. h. in dem Prinzip, welches ihr allein eigen ist". Synästhesie wird also nicht im Sinne beliebiger Austauschbarkeit der Medien propagiert. Auch vom Wert der Logik, der Mathematik, der Konstruktion ist mehrfach die Rede. Die Gründe für solche Widersprüchlichkeit wird man letztlich in Kandinskys psychischer Struktur zu suchen haben. Schon 1903 bekennt der Künstler gegenüber Gabriele Münter, „daß ich jeden, der zu mir nähersteht, unglücklich mache. Das ist mein Schicksal. Daran leide ich selbst. Und dies sind wieder die ,widersprechenden Elemente in mir". Ein anderes Mal klagt er: „Mein Herz fühlt immer verschiedenes zu gleicher Zeit", oder: „Ich habe Sehnsucht - aber wonach? Das weiß ich selbst nicht." Und er offenbart sich in der paradoxen Sprachfigur: „Ich freue mich traurig." Man mag daraus auf eine dualistische Grundverfassung schließen, die auf ihre Weise an die der Romantiker erinnert, aber nur bedingt eine historische Ableitung verträgt. Allenfalls ließen sich Annäherungen der vorgegebenen schizothymen Individualmerkmale an ähnliche Eigenschaften bestimmter historischer Vorläufer unterstellen und Analogien der äußeren Bedingungen aufzeigen. Der antiaufklärerischen Stimmung um 1800 wäre etwa der Antipositivismus und Antimaterialismus der Zeit um 1900 parallelzusetzen und als Nährboden innerer Konflikte bei entsprechend sensiblen Naturen zu interpretieren. Fest steht, daß Kandinskys komplizierte psychische Konstitution die Voraussetzung einer Anfälligkeit für heterogene Einwirkungen bot. Macht des Gefühls und Anspruch des Verstandes, Neigung zur Mystik und Wille zur Organisation, Einsicht in die Isoliertheit des schöpferischen Individuums und Glaube an die universale Mitteilungskraft der ungegenständlichen Farb-Formen-Sprache, missionarischer Eifer im Dienste des neuen abstrakten Idioms und Toleranz gegenüber anderen Ausdrucksweisen: diese gegensätzlichen Elemente waren in Kandinsky mit wechselnder Intensität wirksam. Hinter der oft beschriebenen weltmännischen Sicherheit verbarg sich eine Verletzlichkeit und Zerrissenheit, die den Künstler sogar Zuflucht beim Okkulten suchen ließ und die an der persönlichen Aufrichtigkeit der Hinwendung zum Metaphysischen kaum Zweifel gestattet. Gelegentlich war die Überlagerung einer Komponente durch die gegensinnige die Ursache eigenartiger Mißverständnisse: So führte die Bekanntschaft mit der Atomzerfalls-Hypothese Rutherfords und Soddys bei Kandinsky nicht etwa zu einer bewundernden Verneigung vor dem naturwissenschaftlichen Fortschritt, sondern zu einem mehr erschrockenen als Genugtuung bringenden Verdacht, das Ende des wissenschaftlichen Rationalismus sei gekommen und mit ihm der Anfang des Chaos. Der ausgebildete Wissenschaftler - Kandinsky hatte ja eine Universitätskarriere als Jurist zugunsten der Malerei aufgegeben! - kapitulierte vor den Konsequenzen wissenschaftlichen Denkens, indem er diese Konsequenzen auf ein mit rationalen Kategorien allein nicht faßbares Weltbild zurückbezog. In anderem Zusammenhang rekurriert Kandinsky wieder gerne auf Erkenntnisse der Wissenschaft, dann nämlich, wenn sich Deckungsmöglichkeiten mit den eigenen Vorstellungen anzubieten scheinen oder tatsächlich gegeben sind.

Fragt man nach den konstituierenden Kräften des Weltbildes Kandinskys, so sieht man sich vor allem auf das dritte, „Geistige Wendung" überschriebene Kapitel von „Über das Geistige in der Kunst" hingelenkt. Hier wird zunächst das vorher skizzierte Bild des geistigen Lebens - laut Kandinsky ein "großes spitzes Dreieck in ungleiche Teile geteilt, mit der spitzesten, kleinsten Abteilung nach oben gewendet" - weiter- 
entwickelt und die ohnehin esoterische Gedankenkette um sonderbare Glieder vermehrt. Nach einer ätzenden Abrechnung mit den Positivisten (denen, die nur das erkennen, „was gewogen, gemessen werden kann") folgt ein Lob auf jene wenigen, ,die das sehen können, was von der heutigen Wissenschaft, noch nicht erklärt' wurde". In einem „Reich von morgen" werde Geist nur durch Gefühl („wozu das Talent des Künstlers die Bahn" sei) erkannt werden. Es „vermehren sich oder werden nur öfter bekannt", so ist zu lesen, ,solche Tatsachen, die die gestrige Wissenschaft mit dem gewohnten Wort ,Schwindel' begrüßte. Sogar Zeitungen, diese größtenteils gehorsamsten Diener des Erfolgs und der Plebs, die den Handel mit, was ihr wollt' treiben, finden sich gezwungen, in manchen Fällen den ironischen Ton ihrer Berichte der, Wunder' zu beschränken und auch gar zu unterlassen. Verschiedene Gelehrte, unter welchen sich reinste Materialisten befanden, widmen ihre Kräfte der wissenschaftlichen Untersuchung der rätselhaften Tatsachen, die nicht mehr zu leugnen, nicht mehr zu verschweigen sind". Und nun folgen jene Hinweise auf die Theosophie, auf Helena Petrowna Blavatsky und Rudolf Steiner, die von den Kandinsky-Interpreten wohl niemals übersehen, aber bis in jüngste Zeit nicht in ihrer Tragweite gewürdigt worden sind. Es ist richtig, daß Kandinsky in seinen Schriften mancherlei übernommenes Gedankengut ohne Quellennachweis offeriert; um so bemerkenswerter ist die dem Künstler offenkundig wichtige Nennung der Theosophie und ihrer Anwälte, auch wenn ein skeptischer Passus die zum Ausdruck gebrachte Wertschätzung einschränkt. Es bleibe, so Kandinsky, in jedem Fall die, geistige Bewegung da, welche in der geistigen Atmosphäre ein starkes Agens ist und die auch in dieser Form als Erlösungsklang zu manchem verzweifelten in Finsternis und Nacht gehüllten Herzen gelangen wird ..."

Es ist Sixten Ringboms Verdienst, den theosophischen Momenten in Kandinskys theoretischem und künstlerischem Oeuvre mit Gründlichkeit und Spürsinn nachgegangen zu sein. Man wird wohl fragen müssen, ob Ringboms Ergebnisse, namentlich was die Bildinterpretationen angeht, in vollem Umfang haltbar sind. An der prinzipiellen Richtigkeit des Ansatzes und vieler Resultate wird man nicht mehr vorbei können, zumal eine stattliche Reihe mystischer und theosophischer Bücher im KandinskyMünter-Nachlaß beweist, daß die Berührung mit dem Okkulten durchaus nicht beiläufiger Natur war.

Hatte beispielsweise schon Ettlinger darauf aufmerksam gemacht, daß Kandinsky die Goethesche Vorstellung von der schöpferischen Kraft des Künstlers offenbar in einer durch Steiner stark verfärbten Redaktion vertraut war, derzufolge die Mission des Künstlers in der Transferierung der Welt in Sphären des Geistigen und Göttlichen bestehe, so hat Ringbom die Mittlerfunktion Steiners im Hinblick auf Kandinskys Goethe-Kenntnis energisch unterstrichen. $1884-1897$ hatte Steiner Goethes wissenschaftliche Schriften in Kürschners Deutscher Nationalliteratur ediert, 1888 einen Aufsatz über "Goethe als Ästhetiker" veröffentlicht, der 1909 in neuer und dieses Mal im Geiste der Theosophie modifizierter Form herauskam; 1897 war überdies ein vielbeachteter, aber sehr einseitig abgestützter Artikel über "Goethes Weltanschauung" erschienen.

Die romantisch anmutende Wendung der Auffassung von der künstlerischen Sendung ins Kosmische spielt bei Steiner eine bedeutende Rolle. In Steiners Vorwort der 1907 edierten „Großen Eingeweihten" Edouard Schurés konnte Kandinsky der Überzeugung begegnen, daß Künstler und Hellseher die geheimsten Gesetze des Kosmos 
zu enthüllen berufen seien, konnte er zudem eine leidenschaftliche Polemik gegen Materialismus, Positivismus und Skeptizismus registrieren. Die Lehre von der Reinheit der Kunst und vom unauflösbaren Bund von Kunst, Wissenschaft und Religion im Zeichen einer künftigen Erneuerung (der großen Synthese), die Behauptung der Existenz einer höheren Welt, mit der die Seele kommuniziere wie der Leib mit der materiellen Welt: Alles das bot sich bei Steiner aufs eindringlichste dar. Aber solche allgemeine kulturkritische und adventistische Eröffnungen wären weit weniger interessant, würden sich auf theosophischer Seite nicht Ansatzpunkte für die in engerem Sinne kunsttheoretischen Erwägungen, ja für die künstlerische Praxis Kandinskys erkennen lassen. In "The Key to Theosophy" von H. P. Blavatsky, in "Man Visible and Invisible; Examples of Different Types of Men as Seen by Means of Trained Clairvoyance von C. W. Leadbeater, in "Thought-Forms" von A. Besant und C. W. Leadbeater alles Schriften, die vor 1910 in deutschen Übersetzungen Verbreitung fanden - und schließlich in mehreren Veröffentlichungen Steiners sind okkulte Lehren formuliert, die ästhetische Bereiche tangieren, (ohne freilich auf ästhetische Primärziele ausgerichtet zu sein). Kernvorstellung ist eine komplexe Struktur des menschlichen Körpers: Den sichtbaren Schichten - die Dinge müssen hier sehr vereinfacht dargestellt werden! - sind Zonen übergeordnet, welche sich normalen Sinnen nicht erschließen. In der Astral- und der Mentalzone manifestieren sich Gefühle und Gedanken indessen hellseherischen Blicken als Auren, deren Formen und Farben jeweils bestimmten Bedeutungen entsprechen. Die Emanationen sind, so wird behauptet, auf verschiedene Weise visualisierbar: als durch mediale Fähigkeiten erschlossene Schaubilder oder als sogenannte "Transzendental-Photographien", die man gleichsam als spiritistische $\mathrm{Pa}$ raphrasen auf Röntgenbilder bezeichnen könnte (in der Tat vergleichen Besant und Leadbeater den Pariser Okkultisten Dr. Baraduc mit Röntgen!). Für die Kommunikation sind nach Baraduc und Albert de Rochas Vibrationen des Astralleibes verantwortlich, die von der Seele des Eingeweihten empfangen werden. (In „Über das Geistige in der Kunst" fordert Kandinsky vom guten und starken Kunstwerk, es müsse „rein klingende Seelenvibrationen"e hervorrufen.) Die Gefühls- und Gedankenformen werden von Baraduc als Vibrationsstrukturen veranschaulicht. Besant und Leadbeater lassen ihre Illustratoren eigenartige Figurationen schaffen, die als abstrakte Formeln für etwas wirken, aber als Darstellungen übersinnlich erfahrener Phänomene gemeint sind: „Heftiger Zorn" (ein Ensemble spiraliger und winkelig zuckender Gebilde), ,Jähe "Furcht" (diagonal stiebende Linien und Sichelformen) oder "Geistige Sehnsucht" (ein heller Pfeilbalken vor schwarzem Grund).

Ohne auf theosophische Intentionen und gestalterischen Rang zu achten: Es handelt sich immerhin um Zuordnungen abstrakter Figurationen zu bestimmten Empfindungen, zum ersten Male etwa ein Jahrzehnt vor Kandinskys frühesten ungegenständlichen Arbeiten publiziert! (Übrigens haben vor Ringbom bereits T. H. Robsjohn-Gibbings, Klaus Brisch und andere Autoren auf dieses bemerkenswerte Faktum hingewiesen!) Eine andere Gruppe von Illustrationen zu den „Gedankenformen " Besants und Leadbeaters präsentiert „Forms built by Music"e: Gounod wird mit einer baumkronenartigen Kolonie welliger Linien bedacht, Wagner mit einem Getürme felsähnlich gezackter Formen. Wieder rangiert der Anspruch der Vergegenwärtigung von Unsichtbarem vor der ästhetischen Erwartung, aber das Resultat erscheint doch zugleich als Lösungsvorschlag synästhetischer Probleme. Wobei hinzuzufügen wäre, daß auch die Frage des Farbschmeckens von Theosophen und Okkultisten eifrig diskutiert wurde. 
Berücksichtigt man alle diese Umstände, gewinnen bestimmte Aussagen Steiners ein eigentümliches Gewicht: „Nun aber erwirbt sich der Geheimschüler eben die Fähigkeit, Bilder zu formen, auch wo keine Sinnesgegenstände vorhanden sind. Es muß dann bei ihm an die Stelle des, äußeren Gegenstandes' ein anderer treten. Er muß Bilder haben können, auch wenn kein Gegenstand seine Sinne berührt ... Dies ist die Imagination ... [Die Bilder] sind so lebhaft und wahr wie die Sinnesbilder, nur kommen sie nicht vom Materiellen, sondern vom Seelischen und Geistigen ...“; „Es sind gewisse Linienformen, Gestalten, die man erlebt ... Man lernt erkennen, daß man mit seinem bewegten Ich hineingeflochten ist in die schaffenden Weltenkräfte"; „Alle Dinge werden in ihrem innersten Wesen geistig hörbar."

Konfrontieren wir damit eine Auswahl Kandinskyscher Zitate: „Auf eine geheimnisvolle, rätselhafte, mystische Weise entsteht das wahre Kunstwerk ,aus dem Künstler'. Von ihm losgelöst bekommt es ein selbständiges Leben, wird zur Persönlichkeit, zu einem selbständigen, geistig atmenden Subjekt, welches auch ein materiell reales Leben führt, welches ein Wesen ist. Es ist also nicht eine gleichgültig und zufällig entstandene Erscheinung, die auch gleichgültig in dem geistigen Leben weilt, sondern, wie jedes Wesen besitzt es weiterschaffende, aktive Kräfte. Es lebt, wirkt und ist an der Schöpfung der besprochenen geistigen Atmosphäre tätig." Vom Leitmotiv Richard Wagners heißt es: „Dieses Motiv ist eine Art musikalisch ausgedrückter geistiger Atmosphäre, die dem Helden vorausgeht, die er also auf Entfernung geistig ausströmt." Oder: „Die Form selbst, wenn sie auch ganz abstrakt ist und einer geometrischen gleicht, hat ihren innern Klang, ist ein geistiges Wesen mit Eigenschaften, die mit dieser Form identisch sind“"; „Da kommt aber unfehlbar einer von uns Menschen, der in allem uns gleich ist, aber eine geheimnisvoll in ihn gepflanzte Kraft des ,Sehens' in sich birgt"; schließlich: „Die Welt klingt. Sie ist ein Kosmos der geistig wirkenden Wesen. So ist die tote Materie lebender Geist."

Das sind nicht nur Übereinstimmungen im Tenor, sondern - bei aller Respektierung der verschiedenartigen Akzentsetzung - Gemeinsamkeiten im Substantiellen. Wobei Steiner stets die Wirksamkeit übersinnlicher Kräfte im Sinne hat, Kandinsky die Sprachkraft vom Gegenständlichen losgelöster Farb-Form-Werte.

Manifestieren sich nun die offenkundig theosophisch inspirierten Vorstellungen in Kandinskys Kunst? Da wären gewisse, von Ringbom hervorgehobene Ähnlichkeiten Kandinskyscher Bilder mit Illustrationen aus Besant-Leadbeater, wie die der „Landschaft mit Kirche II" von 1913 mit der genannten Wagner-Musikform. Ich muß gestehen, daß mir die Beziehung der Zackenformation und der überfangenden Bögen zu vordergründig ist, als daß der Vergleich wirklich trüge. Nicht ganz nachvollziehen kann ich überdies Ringboms Deutung verschiedener, mit der 1913 entstandenen Holzschnittserie „Klänge" zusammenhängenden Kompositionen im Sinne der Theosophie. Dagegen bieten einige andere Bilder Ansatzpunkte für eine ebenso interessante wie fruchtbare Argumentation. Daß die 1912 gemalte „Dame in Moskau" zu theosophischer Interpretation verlockt, haben bereits Grohmann und andere Autoren festgestellt. Die weibliche Figur im Zentrum des Bildes ist von einer farbigen Aura umgeben; rechts über ihr schwebt ein unförmiges schwarzes Gebilde, das sich zwischen Frau und Sonne zu schieben im Begriffe ist. Sofern Schwarz für Materie minderen Ranges steht, wäre die Szene als Akt der Bedrohung des weiblichen Wesens, als Einbruch materialistischer Finsternis in die Kommunikationssphäre zwischen ewigem Licht und erleuchtungssuchendem Geschöpf zu lesen; noch weiter ausholend: als Widerstreit zwi- 
schen den lichten Mächten der Liebe und den dunklen des Hasses. Man könnte die Darstellung als okkultistisch-bizarre Eskapade abtun, fänden sich bestimmte Elemente nicht in gleichzeitig oder wenig später entstandenen Bildern wieder, und zwar in weitgehend abstrakten. Es ist bekannt, daß Kandinsky viele seiner ungegenständlichen Werke der Jahre zwischen 1910 und 1914 sehr sorgfältig vorbereitete. Das wie ein jäher, impulsiver Durchbruch anmutende sogenannte „Erste abstrakte Aquarell" ist nach heute weithin akezptierter Auffassung in Wirklichkeit ein (später aus unbekannten Gründen in das Jahr 1910 zurückdatierter) Zwischenentwurf für die Komposition VII von 1913. Man weiß auch, daß die sieben großen Kompositionen der für die Zukunft der Malerei so entscheidenden Jahre 1910 bis 1914 von figurativen Keimen aus entfaltet worden sind. Der Abbau des Gegenständlichen vollzog sich dabei auf doppeltem Wege: einmal durch das Eindringen ungegenständlicher Elemente ins Bild, zum anderen durch die fortschreitende Reduktion der gegenständlichen Elemente selber. Gelegentlich sind in die Finalfassung Gegenstandsreminiszenzen zurückgeschleust, die in den Zwischenstufen bereits überwunden scheinen. Das Verfahren zeugt übrigens von erstaunlichem bildnerischem Kalkül. Werke, die in ihrer formalen und koloristischen Vehemenz an die berühmten Sätze denken lassen: „Kampf der Töne, das verlorene Gleichgewicht, fallende ,Prinzipien, unerwartete Trommelschläge, große Fragen, scheinbar zielloses Streben, scheinbar zerrissener Drang und Sehnsucht, zerschlagene Ketten und Bänder, ... Gegensätze und Widersprïche - das ist unsere Harmonie", Werke also, die solche Empfindungen glaubhaft verbildlichen, sind durch Skizzen vorbereitet, welche in ihrer knappen Präzision auf die Arbeiten der zwanziger Jahre vorausweisen. In der Tat: „inszenierte Vorläufigkeit", aber - wie ich meine - aus einer Widersprüchlichkeit geboren, der es nicht einfach um die Verhüllung der Ratio um eines bestimmten Effektes willen geht.

Kehren wir zurück zum schwarzen Fleck der „Dame in Moskau". Frappierenderweise ist dieses Bildelement Thema eines „Schwarzer Fleck I" betitelten Ölbildes (Leningrad, Russisches Museum), das kurz nach der „Dame in Moskau" gemalt und durch Kompositionsskizzen präpariert wurde. $\mathrm{Daß}$ ein über das Fleckmotiv hinausgehender Zusammenhang besteht, wird durch das Nebenmotiv der Kutsche bewiesen. Auf der „Dame in Moskau" ist das Vehikel hinter der Frauenfigur zu erblicken; auf dem Leningrader Gemälde und auf einem Kompositionsentwurf in München erscheint es freischwebend, der Realfunktion entbunden. Der schwarze Fleck selber ist einer sonnenartigen Insel rechts oben konfrontiert und zu einer Art losgelöster Anti-Aura geworden. Ein Holzschnitt der Serie „Klänge" (1913) wiederholt die Konstellation; von der Kutsche ist ein Kürzel übriggeblieben.

Die kühnste Konsequenz, die man aus diesen von Ringbom gemachten Beobachtungen ziehen könnte, wäre diese: Da sich bestimmte abstrakte Formen auf Bildern der Geniezeit Kandinskys von definierbaren, und zwar im theosophischen Sinne definierbaren, Figurationen ableiten lassen, ist die Summe der abstrakten Formen ebenfalls auf theosophische Emanations- und Vibrationsvorstellungen zurückzubeziehen. Nun, Ringbom versteigt sich nicht zu dieser Behauptung, wie er denn überhaupt nicht versucht, das theosophische Engagement Kandinskys zu verabsolutieren. Aber er ist doch geneigt, eine größere Zahl von Bildern vom erläuterten Ansatz aus zu erklären und die zunehmende Befreiung der Formen und Farben von Gegenstandsbindungen als eine Autonomisierung analog der Steinerschen Idee, „daß das Geistige ganz unmittelbar in den freischwebenden Tönen, Farben usw. sich auslebt", zu deuten. Nun wird man 
manchem der zwischen 1910 und dem Ersten Weltkrieg entstandenen Arbeiten den Charakter des Schwebend-Bewegten zugestehen können; die Mehrzahl wird indessen durch die vorhin bereits erwähnte Turbulenz gekennzeichnet. Um noch einmal Kandinsky zu zitieren: „Die Stimmgabel war aber ,Dramatik'. Explosionen, zusammenprallende Flecke, verzweifelte Linien, Ausbruch, Dröhnen, Auseinanderfliegen - Katastrophen. Die sämtlichen Elemente, die Konstruktion und selbst die technische Art bis zum einzelnen Pinselstrich waren diesem Zweck, Dramatike untergeordnet. Verlorenes Gleichgewicht, aber kein Untergang."

Es scheint nicht anzugehen, diese Farb-Form-Dramen auf jeweils deutbare Einzelinhalte zu befragen, auch dann nicht, wenn sie in der Deszendenz ikonographisch mehr oder minder bestimmbarer Darstellungen stehen. In Sonderfällen, wie dem „Schwarzen Fleck", sind ohne Zweifel echte Dechiffrierungschancen gegeben. Generell allerdings hat man, so möchte ich meinen, Formen und Farben nicht als genauer ortbare Bedeutungsträger aufzufassen, vielmehr als freie Fügungen mit Hinweischarakter. Daß die Grundvorstellung einer Vermittelbarkeit von Gefühlen und Gedanken durch visuelle Medien dabei auf eine von der Theosophie stimulierte Weise zum Tragen kommt, dürfte nach den Darlegungen Ringboms gesichert sein.

Für die Werke der „Kühlen Periode“, also der Zeit seit etwa 1920, gelten zweifellos komplexere Bedingungen. Gewiß bezieht sich Kandinsky in „Punkt und Linie zu Fläche" (1926) noch einmal auf die "Gedankenformen" von Besant und Leadbeater, gewiß bieten sich Vergleiche zwischen Bildern wie "Weiße Schärfe" von 1928 und dem „Upward Rush of Devotion" im genannten Werk der beiden englischen Theosophen an. Aber für die Bildlösungen dieser zweiten Reifephase spielen die Auseinandersetzungen mit Neoplastizismus, Suprematismus und Konstruktivismus eine wichtige Rolle; und überdies wird man der Gestaltpsychologie eine bestimmte Wirkung einräumen müssen. Wahr ist allerdings, daß Kandinskys Einstellung zu den geistigen Prämissen seines Tuns trotz aller Bemühungen ums Systematische und Analytische in wesentlichen Bereichen eigentümlich ungebrochen bleibt. Die Zuversicht, eine neue Ära der für geistige Erfahrungen Sensibilisierten werde heraufziehen, und der Glaube an die Dominanz des Prinzips der inneren Notwendigkeit beherrschen nach wie vor die Vorstellung des Künstlers, so daß man Ringboms auf diese Periode gemünzte Behauptung, es wandelten sich nur die Mittel, nicht die Ziele, mit den angemessenen Einschränkungen akzeptieren darf.

Kehren wir noch einmal zu den Bildern der dynamisch-expressiven Phase zurück und rufen wir uns das zitierte: „Verlorenes Gleichgewicht, aber kein Untergang" ins Gedächtnis. Eschatologische Gedanken sind in manchem Bildtitel der Jahre zwischen 1910 und 1914 lebendig (es seien nur "Sintflut", ,Jüngster Tag", „W iederauferstehung" genannt), und im „Rückblick" lesen wir: „Ein großer, objektiv wirkender Untergang ist ebenso ein vollständig und im Klang abgetrennt lebendes Loblied, wie ein Hymnus der neuen Entstehung, die dem Untergang folgt" (Interpretation zu "Komposition 6"); und, im Zusammenhang mit der Forderung nach der Gültigkeit des ,inneren Lebens der Werke": „Hier bemerkte ich zu meiner Überraschung, daß diese Forderung auf der Basis gewachsen ist, die Christus als eine moralische Qualifikationsbasis aufstellte. Ich bemerkte, daß diese Kunstanschauung christlich ist und daß sie zu derselben Zeit die nötigen Elemente zum Empfang der ,dritten' Offenbarung, der Offenbarung des Geistes, in sich birgt." Das sind, wie man zu Recht festgestellt hat, joachitische (oder besser: neojoachitische) Gedanken, wie sie sich modifiziert auch 
in der theosophischen Lehre vom Anbruch der Ära des Geistigen manifestieren. Aber in der direkten Qualifizierung der Kunstanschauung als christlich wird eine andere Quelle der Haltung Kandinskys erkennbar: die (zwar nicht im Sinne von Kirchentreue bestehende, aber doch lebenslang formell aufrecht erhaltene) Bindung an den christlich-orthodoxen Glauben. Von hier aus läßt sich nun eine Brücke schlagen, die zum Ausgangspunkt unserer Überlegungen zurückführt.

Einmal spielt der joachitische Gedanke einer spiritualistischen Erneuerung seit Lessings "Erziehung des Menschengeschlechts" eine beachtliche Rolle in der deutschen Philosophie und Literatur um 1800. Lessing hatte das Modell einer stufenweisen Erziehung der Menschheit mit der Vorstellung des Joachim von Fiore verbunden, das neutestamentliche Christentum werde sich progressiv in ein Christentum des Geistes verwandeln. Bei Schelling, Fichte und Hegel finden sich Reflexe und Varianten dieser Konstruktion. Runge meditiert über eine durch abstrakten Geist geprägte, aber nichtsdestoweniger innige Religion, der eine adäquate Kunst entspringen müsse. Das neunzehnte Jahrhundert hindurch bleiben spiritualistische Ideen letztlich joachitischer Provenienz lebendig, um im Symbolismus, namentlich im russischen, neue Früchte zu tragen. Auf welchem Wege die Vorstellungen Kandinsky erreicht haben, ist ungewiß; der Gedanke an Mereschkowsky liegt wohl näher als der an die deutsche Romantik.

Mit den Malern der deutschen Frühromantik teilt Kandinsky indessen bis zu einem gewissen Grade ein seinerzeit von Lankheit beschriebenes Schicksal. Nämlich zwischen traditionellem Christentum und einer Haltung, welcher Kunst ein autonomes Medium des Weltverständnisses bedeutet, entscheiden zu müssen. Man weiß, daß die Entscheidung bei Friedrich und Runge unausgetragen blieb und daß gerade der Konflikt die spezifischen Möglichkeiten und Grenzen ihres Schaffens bestimmte. Ob primär an diesem Dualismus der Drang nach Befreiung vom Gegenständlichen scheitern mußte, ist freilich sehr die Frage. Für Kandinsky stellte sich das Problem ganz gewiß nicht mit einer der Situation um 1800 vergleichbaren Intensität. Immerhin bezeugt das zitierte Legitimierungsbemühen, daß Auseinandersetzung mit der Tradition für ihn auch Konfrontation mit dem christlichen Glauben hieß. Die historisch möglich gewordene Entscheidung zugunsten der Verabsolutierung der Formensprache durch joachitisch-spiritualistische Überlegungen abzudecken, kann nur die Richtigkeit dieser Behauptung bekräftigen.

Wir sind an dem Punkte, wo es aus den dargelegten Gedanken eine Summe zu ziehen gilt. Eine schlüssige Antwort wird niemand erwarten, eher ein unbequemes und unvollständiges Bündel von Einzelantworten.

Überzeugt bin ich, daß man Kandinskys Welt- und Kunstverständnis im Sinne eines absolut redlichen persönlichen Synkretismus' zu begreifen hat. Der synkretistische Charakter ist wesentlich durch die besondere Veranlagung bedingt, die den Künstler für Anregungen heterogener Art empfänglich machte und zugleich jeder harmonischen Legierung im Wege stand. Synästhetische Fähigkeiten und eine Neigung zur sektiererischen Übersteigerung kommen als konstitutionelle Faktoren hinzu. Die derart beschaffene Persönlichkeitsstruktur läßt Kandinsky manchen Repräsentanten der Romantik verwandt erscheinen. Über die prinzipielle Affinität hinaus sind, wie erläutert, mehrere Möglichkeiten einer Begegnung mit romantischem Gedankengut in Rechnung zu stellen. Aber angesichts des Mangels schlüssiger Belege für Direktkontakte hat man manches, was sich als Resultat unmittelbarer Beziehung ausnimmt, vorsichtshalber im Sinne von Analogien oder von Übernahmen nach längeren Überliefe- 
rungsprozessen zu werten. Außer Frage steht nach den Forschungen von Ringbom die Bedeutung theosophischer Vorstellungen für Kandinskys Denkgebäude. Für die künstlerische Praxis wird man indessen die theosophischen Einflüsse meines Erachtens nicht überschätzen dürfen. Wenngleich sich in Einzelfällen bestimmte Linien verfolgen lassen, weist die Befreiungstat Kandinskys über alle okkultistische Programmatik hinaus. Die Einsetzung einer Sprache zur Vermittlung figurativ nicht artikulierbarer geistiger und emotionaler Botschaften ist zwar auch ein Akt der Codierung, vor allem aber ein solcher der gestalterischen Emanzipation. Insofern scheint der in Tiecks an die Maler gerichtete Vorwurf: „Aber euch fehlen Farben, und Bedeutung im gewöhnlichen Sinne ist leider die Bedingung eurer Kunst"e, scheint also die in dieser Vorhaltung eingeschlossene Aufforderung zur Erkundung neuer bildnerischer Wege von Kandinsky umfassend und überzeugend eingelöst.

In Anbetracht der Tatsache, daß es sich um den Abdruck eines unveränderten Vortragsmanuskriptes handelt, wurde auf einen Anmerkungsapparat verzichtet. Der Leser sei auf folgende Schriften hingewiesen:

W ASSILY KandinSKY, Über das Geistige in der Kunst, München 1912 (Ausgabe von MaX BiLl, BernBümpliz 1952 ${ }^{4}$ ). - W ASSILY KANDINSKY, Rückblick, Berlin 1913 (Ausgabe von LudWIG Grote, BadenBaden 1955). - W ASsily KANDINSKY, Punkt und Linie zu Fläche, München 1926 (Ausgabe von MAX BILL, Bern-Bümpliz 1964 ${ }^{5}$ ). - W AssiLY KANDINSKY, Essays über Kunst und Künstler, Stuttgart 1955 (Ausgabe von MAX BiLL). - JohANNES EICHNER, Kandinsky und Gabriele Münter. Von den Ursprüngen moderner Kunst, München 1957.- L. D. ETTLinger, Kandinsky's „At Rest", London 1961 (Charlton Lectures on Art). - Friedhelm W. Fischer, Geheimlehren und moderne Kunst, in: Fin de Siècle. Zu Literatur und Kunst der Jahrhundertwende, Frankfurt am Main 1977, S. 344 ff. - W ILL GrohmanN, Wassily Kandinsky - Leben und Werk, Köln $1961^{2}$ (mit umfangreicher Bibliographie). - Werner HofManN, Grundlagen der modernen Kunst, Stuttgart 1966. - Klaus LANKHerT, Die Frühromantik und die Grundlagen der "gegenstandslosen" Malerei, in: Neue Heidelberger Jahrbücher 1951, S. 55 ff. - PAUL OVERY, Kandinsky. Die Sprache des Auges, Köln 1969. - Peter Anselm RiedL, Abstrakte Kunst und der Traum von der rezeptiven Gesellschaft, in: Festschrift für Klaus Lankheit, Köln 1973, S. 67 ff. - SiXTEN RINGBOM, Art in the "Epoch of the Great Spiritual": Occult Elements in the Early Theory of Abstract Painting, in: Journal of the Warburg and Courtauld Institutes, XXIX, 1966, S. 386 ff. - SiXteN R INGBOM, The Sounding Cosmos. A Study in the Spiritualism of Kandinsky and the Genesis of Abstract Painting, Abo 1970 (mit umfangreichem Literatur- und Quellenverzeichnis, besonders auch zu den Themen Theosophie und Okkultismus). - OTto STEıZER, Die Vorgeschichte der abstrakten Kunst. Denkmodelle und Vor-Bilder, München 1964.

Die im Text genannten Kunstwerke sind bei Stelzer und Ringbom reproduziert. In einer vom Verfasser für den Rowohlt-Verlag vorbereiteten Kandinsky-Monographie werden die hier skizzierten Probleme ausführlicher erörtert werden. 